most common cyanotic congenital heart defect. The causes of congenital heart disease are poorly understood. Histone modifying genes have previously been implicated in congenital heart disease. KMT2C is a histone modifying gene which contains a catalytic SET domain which methylates histone 3 lysine 4 regulating gene expression. We have previously identified 18 mutations in KMT2C in a whole-exome sequencing study of 829 non-syndromic tetralogy of Fallot patients. These mutations are absent in gnomAD and had a combined annotation dependent depletion (CADD) score of more than 20 indicating the variants are rare and likely deleterious. We have now investigated the role of this gene further in tetralogy of Fallot and heart development. KMT2C expression during heart development has been studied using RT-PCR and in situ hybridisation. $\mathrm{Kmt} 2 \mathrm{c}$ is expressed throughout the mouse embryonic heart from embryonic day 11.5 to 14.5 which is the key developmental time period for heart development with respect to the defects observed in tetralogy of Fallot. In human embryonic hearts cDNA expression of KMT2C was also found between Carnegie stages 13 and 20 (equivalent stages to mouse E11.5-14.5). Heart defects have been characterised using a mouse model where the SET domain of KMT2C has been deleted resulting in a catalytically inactive protein being produced. In mouse embryos homozygous for the deletion, all embryos appear to have abnormal heart development and ventricular septal defects with or without an overriding aorta (found in seven out of ten embryos studied, shown in figure) are the most common defect indicating similarities to tetralogy of Fallot. Other defects seen in these hearts have included abnormal ventricular myocardium with resemblance to non-compaction and atrial septal defects. Overall this work demonstrates that KMT2C is a good candidate gene for tetralogy of Fallot, both due to its cardiac expression and the defects exhibited in mice where the SET domain is deleted.

Conflict of interest None

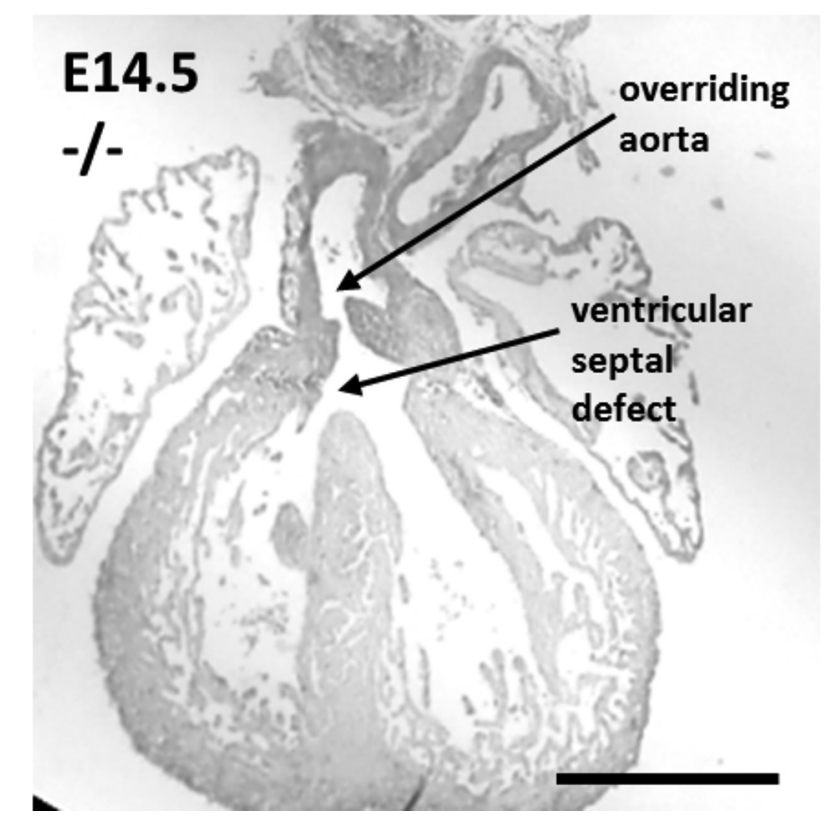

Abstract BS9 Figure 1

\section{BS10 CANAGLIFLOZIN, AN SGLT2 INHIBITOR ATTENUATES ISCHAEMIA/REPERFUSION INJURY IN THE NON- DIABETIC HEART}

Ven Gee Lim, Sapna Arjun, Robert Bell, Derek Yellon. University College London

\subsection{6/heartjnl-2019-BCS.174}

Introduction Sodium-glucose linked cotransporter 2 (SGLT2) inhibition has been shown to have unexpected cardiovascular benefits in recent landmark outcome trials. We previously hypothesized that this is mediated through attenuation of ischaemia/reperfusion injury and have demonstrated significant attenuation of infarct size in both diabetic and non-diabetic animals, irrespective of glucose lowering. That the protection is not found in isolated heart led us to hypothesize that the cardioprotection is via a hormone-mediated mechanism that leads to receptor activated kinase activation and cytoprotection.

Methods Non-diabetic male Sprague-Dawley rats were fed either standard chow or canagliflozin-fortified diet for 24 hours. Blood samples were obtained (glucose, insulin, glucagon, $\beta$-hydroxybutyrate) before the hearts were harvested for Langendorff perfusion, subjected to $35 \mathrm{~min}$ regional ischaemia and either $5 \mathrm{~min}$ or 2 hour reperfusion for either signaling (Western Blot analysis) or infarct size assessment.

Results $24 \mathrm{~h}$ oral canagliflozin led to a significant attenuation in myocardial infarct size (Control $62 \pm 5 \%$ vs Canagliflozin $45 \pm 4 \%, p=0.016)$, equivalent to that found following ischaemic conditioning (IPC, figure 1). Concomitant with this, we found that within the ischaemic myocardium (left ventricle [LV]), there was a significant increase in Akt and ERK (but not STAT3 phosphorylation), an augmentation absent in remote (right ventricular $[\mathrm{RV}]$ ) myocardium (figure 2). Despite maintenance of serum glucose, there was no difference in circulating insulin or glucagon. Similarly, there was no significant chance in the ketone, $\beta$-hydroxybutyrate, suggesting that increased availability of ketones is an unlikely mechanism of cardioprotection in this model.

Conclusion Canagliflozin led to a reduction in myocardial infarct size with associated Akt and ERK phosphorylation (well-known mediators of cardioprotection). Pathway-specific inhibitors may help elucidate the causal role of Akt and ERK activation. Interestingly, circulating levels of insulin, glucagon,

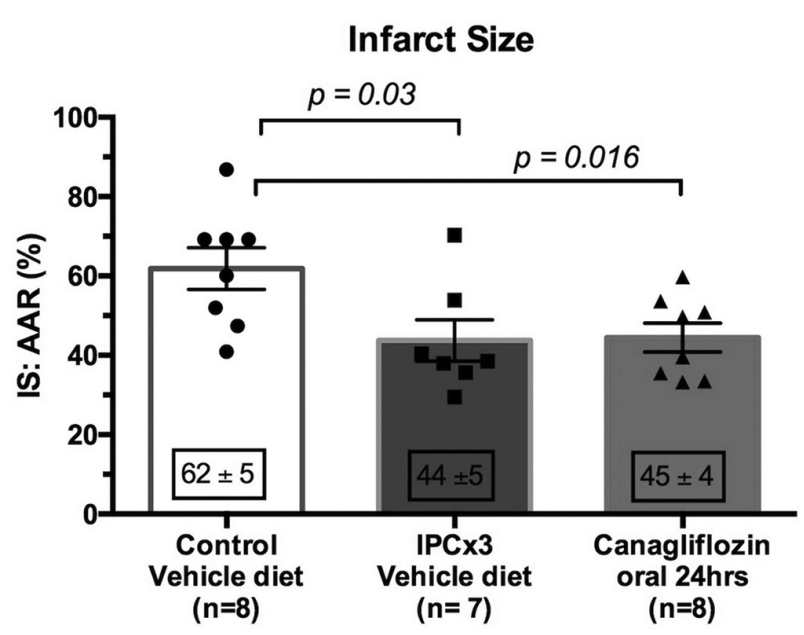

Abstract BS10 Figure 1 